\title{
El rol de las revistas científicas estudiantiles en la difusión de conocimientos en pregrado
}

\author{
The role of student scientific journals in the \\ dissemination of knowledge in undergraduate programs \\ o papel das revistas científicas estudantis na \\ divulgação do conhecimento na graduação
}

\author{
Carlos Miguel Ríos-González * \\ Aarón Eduardo Carvajal-Tapia **
}

\section{Sra. Editora:}

En primera instancia permítannos agradecer por la oportunidad de manifestar nuestras inquietudes respectos al rol de las revistas científicas estudiantiles a través de una de las revistas prestigiosas que viene ampliando sus horizontes de difusión de la investigación científica. Asimismo, felicitar al equipo editorial por la labor que desarrolla en procura de ampliar y colaborar en la consolidación de revistas latinoamericanas, que buscan expandirse y afianzar sus procesos de indización con el objeto de acercarse a revistas prestigiosas de contexto internacional.

Por otra parte, es pertinente recordar que las revistas científicas se establecen como el principal órgano de difusión científica y de conocimientos implícitos (1), el tiempo invertido en la lectura de un manuscrito, la sacrificada dedicación en la revisión, edición y posterior publicación, lo convierten en un invaluable motor de producción de conocimientos en la comunidad científica. Las universidades vienen cumpliendo un doble rol al ser motivadores en la edición de revistas científicas y por otra parte convertirse en consumidores de la producción científica a través de la utilización de la principal fuente de la materia prima producida por las revistas como son los artículos científicos, de esta manera, las universidades son las comprometidas de refrescar el saber humano y brindar los conocimientos ineludibles para orientar el rumbo de la sociedad; sin embargo, en muchas ocasiones existen diversas falencias en este ámbito que en la respectivas instituciones que no se hace énfasis en la potencialización de estos roles $(2,3)$.

De igual manera, resulta importante identificar el impacto de las revistas científicas en la difusión de conocimientos en pregrado, uno de ellos es haber motivado la creación de una serie de Congresos Científicos Nacionales e Internacionales. De una manera loable, en la que estudiantes de pregrado han sido protagonistas (ganadores de congresos, ponentes, autores y editores) con maestros y grandes eminencias en la medicina científica, permitiendo el avance y progreso de nuestra Alma Mater desde 1986, para que se generaran las bases que permitieran la creación de la Federación Latinoamérica de Sociedades Científicas de Estudiantes de Medicina (FELSOCEM) en Valparaíso, Chile (3). Este factor de impacto es medido a través de las citas a la revista, al autor o al artículo en cuestión, jugando un papel fundamental en esto la cantidad de indexaciones que posea la revista, siendo no solo importante esto último para los autores sino también para las revistas.

Si bien este proceso no es nada fácil, la meta de indexar, cumplir criterios y lograr ser una revista de alto impacto conlleva una dedicación, pasión y perseverancia que hoy por hoy se traduce en el nacimiento de nuevas revistas científicas. La colaboración reciproca con otras que mantienen su continuidad ha hecho que las revistas científicas latinoamericanas sean consideradas una corriente muy potente de investigación en contraste con otras regiones del mundo (3-4). De otra parte, muchas de estas revistas

Estudiante de Medicina, Facultad de Ciencias Médicas, Universidad Nacional de Caaguazú, Coronel Oviedo, Paraguay, Sociedad Científica de Estudiantes de Medicina de la Universidad Nacional de Caaguazú, Coronel Oviedo, Paraguay.

** Estudiante de Medicina, Facultad de Medicina, Universidad Mayor de San Andrés, La Paz, Bolivia, Sociedad Científica de Estudiantes de Medicina de la Universidad Mayor de San Andrés, La Paz, Bolivia. 
están indexadas en distintas bases de datos biomédicas, ya sea de manera local, regional e incluso internacional; sin embargo, existe el peligro de que estas revistas de carácter formativo sean aniquiladas por grandes empresas que manejan servicios de indexación con altas fuentes de financiación donde las revistas con escasos recursos presentan problemas para ingresar a Servicios de Indexación y Resumen (SIR) de reconocimiento internacional entre ellas SCI, SSCI, Scopus $(4,5)$.

Bajo estas consideraciones, es importante recordar el rol que cumplen las revistas científicas en la formación de pioneros de la ciencia, que con sus primeras publicaciones se han hecho protagonistas de esto que tanto apasiona el "Hacer Ciencia". El aportar trabajos de investigación han llevado al estudiante a tomar una actitud tenaz, curiosa, un estudiante de pensamiento crítico, innovador, creativo, poco satisfecho de lo mínimo y soñador de lo inconcebible.

Tras esto y recordando las frases de Alexander Fleming: "No son los vestíbulos de mármol los que proporcionan la grandeza intelectual, sino el alma y el cerebro del investigador". Si bien el investigador sufre las decepciones en la preparación de un primer trabajo, el largo tiempo, los fracasos, pero, los fracasos son también útiles, porque bien analizados, conducen al éxito. Las revistas científicas toman este pensamiento y plasman estas sagradas palabras en una publicación de un trabajo exhaustivo de estos actores que van a la vanguardia de la construcción de nuevos conocimientos.
Con todo este trabajo, estamos convencidos de que estos esfuerzos no son en vano y que a través del tiempo este sueño y perdurará, cosechando logros y metas cumplidas, rompiendo fronteras en una magna difusión de conocimientos concebidos, sabiendo que en latinoamérica se dejaron huellas históricas a seguir; sin embargo, existe un riesgo debido a la exigencia del mercado actual con los procesos de indexación internacional, donde los estándares de calidad son mayores, en lo cual algunas revistas se encuentra en desventaja por su carácter netamente formativo y altruista.

\section{Referencias.}

1. Ramírez D, Martínez L, Castellones O. Divulgación y Difusión del Conocimiento: Las revistas Científicas. Bogotá: editorial Universidad Nacional de Colombia; 2012.

2. Estigarribia M. La revista científica como instrumento de difusión de la investigación en las universidades latinoamericanas. UFSC 2012: 11-14.

3. Rios-González CM. El papel de las revistas científicas estudiantiles en la difusión de hallazgos en pregrado. Revista Hispanoamericana de Ciencias de la Salud. 2016; 2 (1): 103.

4. Cabrera-Samith I, Oróstegui-Pinilla D, Angulo-Bazán Y, MaytaTristán P, Rodríguez-Morales AJ. Revistas científicas de estudiantes de medicina en Latinoamérica. Rev Med Chile 2010; 138: 1451-1455.

5. Rodríguez E, Naranjo S, González DL. Publindex: más que un proceso de indexación. El Ágora USB. 2015; 15 (1): 29-41. 